\title{
Synthesis of 4,4-Difluoro-1H-pyrazole Derivatives
}

\author{
Jessica R. Breen ${ }^{a}$ \\ Graham Sandford*a \\ Bhairavi Patel ${ }^{b}$ \\ Jonathan Fray \\ a Department of Chemistry, Durham University, South Road, Durham, \\ DH1 3LE, UK \\ Graham.Sandford@Durham.ac.uk \\ ${ }^{b}$ Pfizer Global Research \& Development, Ramsgate Road, Sandwich, Kent, \\ CT13 9NJ, UK
}

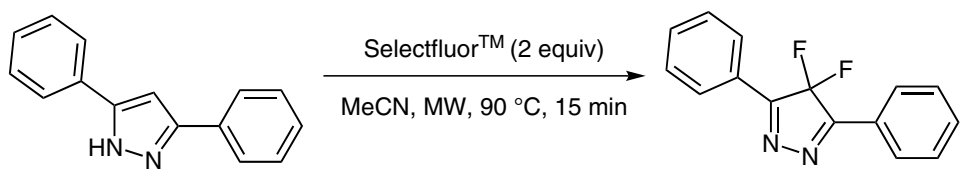

Received: 22.09 .2014

Accepted after revision: 10.10.2014

Published online: 05.11 .2014

DOI: 10.1055/s-0034-1378915; Art ID: st-2014-d0791-c

Abstract Fluorination of 3,5-diarylpyrazole substrates by Selectfluor ${ }^{\mathrm{TM}}$ in acetonitrile gave 4,4-difluoro- $1 \mathrm{H}$-pyrazoles in addition to 4-fluoropyrazole derivatives. The structure of this new class of fluorinated heterocycle was established by X-ray crystallography.

Key words organofluorine, fluoroheterocycle, pyrazole, selective fluorination, fluoropyrazole

The importance of fluorine-containing aromatic and heterocyclic motifs to the pharmaceutical and agrochemical industries continues to grow ${ }^{1}$ because approximately $5-$ $15 \%$ of the total number of drugs launched worldwide over the past 50 years bear fluorinated substituents. ${ }^{2}$ For example, many six-membered fluorinated heteroaromatic derivatives find applications in a wide variety of drugs and plant protection agents such as Xeloda (anticancer, Roche), Voriconazole (antifungal, Pfizer), Ancobon (antifungal, Valeant) and Diclosulam (herbicide, Dow Agroscience). ${ }^{2}$

Whilst there are many reported examples of the synthesis of commercially important fluorinated six-membered azaheterocyclic rings, processes for the preparation of related fluorinated five-membered ring systems are relatively rare. ${ }^{3}$ However, interest in fluoropyrazole derivatives has increased recently due to their potential use for treating diabetes, ${ }^{4}$ inflammatory disease, ${ }^{5}$ as gastric acid inhibitors ${ }^{6}$ and as acaricides. ${ }^{7}$ Consequently, protocols for the synthesis of a variety of selectively fluorinated pyrazoles have been reported using either fluorination or 'fluorinated building block' strategies. Fluorocyclocondensation reactions involving enamino ketones ${ }^{8}$ and fluorocyanoketones, ${ }^{9}$ gold-catalysed aminofluorination of alkynes ${ }^{10}$ and reaction of hydrazines with fluoro- $\beta$-dicarbonyl substrates ${ }^{11}$ offer efficient routes to various functional fluoropyrazole derivatives. Adaptation of established fluorination methodology such as halogen exchange ${ }^{12}$ or Balz-Schiemann processes ${ }^{13}$ has had limited success for the synthesis of fluoropyrazoles from appropriately functionalised pyrazole substrates due to low total yields over several synthetic steps. Potentially, the most efficient methods for the synthesis of fluoropyrazole systems are aromatic substitution processes using electrophilic fluorinating agents. A few examples of the preparation of various fluoroaminopyrazole systems from the reaction of aminopyrazole precursors with NFSI or Selectfluor ${ }^{\mathrm{TM}}$ have been recorded ${ }^{14}$ whilst several 4-fluoropyrazole derivatives have been prepared by reaction of Selectfluor ${ }^{\mathrm{TM}}$ with a range of $\mathrm{N}$-arylpyrazole substrates. ${ }^{15}$

As part of a wider research programme concerning the synthesis of fluoroorganic systems using electrophilic fluorinating agents, ${ }^{16}$ we were interested in broadening the scope of 'late-stage' fluorination reactions of pyrazole derivatives for applications in the life-sciences industries. In this paper, we describe electrophilic fluorination reactions of various pyrazole derivatives with either Selectfluor ${ }^{\mathrm{TM}}$ or fluorine gas which led to the unexpected synthesis of novel 4,4-difluoro-1H-pyrazole systems.

Pyrazole substrates 1 were either obtained from commercial suppliers or synthesised by reaction of the appropriate diketone derivatives with hydrazine or phenyl hydrazine by heating to reflux in ethanol following literature procedures. ${ }^{17}$

We began our pyrazole fluorination studies by investigating reactions of representative pyrazole systems 1a-c with either Selectfluor ${ }^{\mathrm{TM}}$ or fluorine gas and the results are collated in Table 1. Reactions involving Selectfluor ${ }^{\mathrm{TM}}$ were carried out by heating the reaction mixture using microwave irradiation (conditions A). Fluorine gas, diluted to a $10 \%$ mixture in anhydrous nitrogen was passed at a controlled rate via a mass flow controller into a stirred solution of the substrate in acetonitrile using equipment discussed previously (conditions B). ${ }^{16}$ Monofluorinated pyrazoles 2ac were formed in modest yields and could be purified by column chromatography on silica gel. In contrast, fluorination of 3,5-dimethyl-1H-pyrazole was inefficient because of extensive tar formation due to competing fluorination of the pendant methyl substituents and subsequent product 
degradation. In addition, pyrazole systems bearing two electron-withdrawing groups $\left(\mathrm{CF}_{3}, \mathrm{CO}_{2} \mathrm{H}, \mathrm{CO}_{2} \mathrm{Me}\right)$, did not give any observable products upon reaction with either Selectfluor ${ }^{\mathrm{TM}}$ or fluorine gas, reflecting the lower nucleophilicity of these substrates, and starting materials were recovered in all of these reactions.

Table 1 Synthesis of Monofluoropyrazoles 2 using Selectfluor ${ }^{\mathrm{TM}}$ or Fluorine Gas
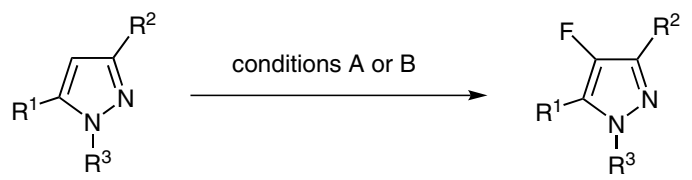

\begin{tabular}{|c|c|c|c|c|c|}
\hline Pyrazole 1 & $\mathrm{R}^{1}$ & $R^{2}$ & $\mathrm{R}^{3}$ & Conditions ${ }^{\mathrm{a}, \mathrm{b}}$ & $\begin{array}{l}\text { Fluoropyrazole } \mathbf{2} \text {, } \\
\text { yield (\%) }\end{array}$ \\
\hline $1 a$ & Me & $\mathrm{Ph}$ & $\mathrm{H}$ & $\begin{array}{l}\text { A } \\
B\end{array}$ & $\begin{array}{l}2 a, 33 \\
2 a, 45\end{array}$ \\
\hline $1 b$ & $\mathrm{CF}_{3}$ & $\mathrm{Ph}$ & $\mathrm{H}$ & $\begin{array}{l}A \\
B\end{array}$ & $\begin{array}{l}\mathbf{2 b}, 43 \\
\mathbf{2 b}, 46\end{array}$ \\
\hline 1c & Me & Me & $\mathrm{Ph}$ & $\begin{array}{l}A \\
B\end{array}$ & $\begin{array}{l}\text { 2c, } 43 \\
\text { 2c, } 40\end{array}$ \\
\hline
\end{tabular}

a Conditions A: Selectfluor ${ }^{\mathrm{TM}}$ (1 equiv), MW, $15 \mathrm{~min}, 90^{\circ} \mathrm{C}$

${ }^{b}$ Conditions B: $10 \% F_{2} / N_{2}$, MeCN, r.t.

Monofluoropyrazole systems 2a-c were identified by NMR and mass spectrometry techniques. In particular, the ${ }^{19} \mathrm{~F}$ NMR spectra of these systems display singlet resonances at approximately $\delta=-175 \mathrm{ppm}$, consistent with reported spectroscopic data reported for related fluoropyrazole systems. ${ }^{15}$ In addition, the structure of $\mathbf{2} \mathbf{b}$ was confirmed by X-ray crystallography (Figure 1$).{ }^{18}$

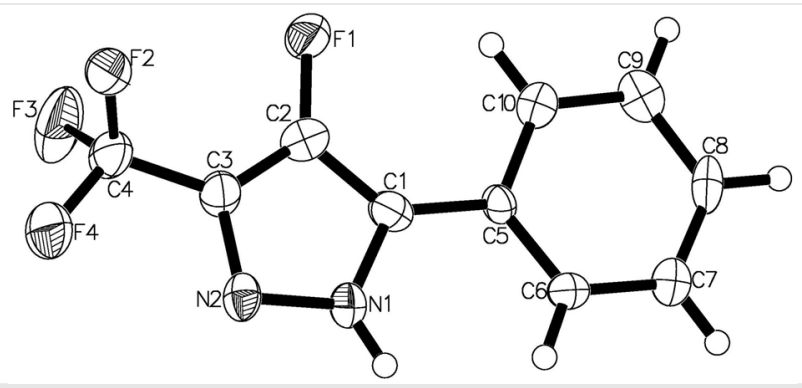

Figure 1 Molecular structure of $\mathbf{2 b}$

In order to expand the scope of the fluorination reactions we studied reactions of diphenylpyrazole substrates 1d-k which unexpectedly gave mixtures of mono- and difluorinated systems $\mathbf{2 d - k}$ and $\mathbf{3 a - h}$ even when only one equivalent of Selectfluor ${ }^{\mathrm{TM}}$ was used and these results are collated in Table 2 (Conditions A). In all reactions, separation and purification of the difluorinated products $\mathbf{3 a}-\mathbf{h}$ were readily achieved because, in general, they eluted from the silica gel column much more rapidly than the starting material and monofluorinated pyrazole systems. Separation of monofluoropyrazole products from the corresponding starting materials proved to be very difficult but could be achieved in several cases. Yields of the 4,4-difluoro- $1 H$-pyrazole products $\mathbf{3 a}-\mathbf{h}$ were improved upon reaction of the pyrazole substrates with two equivalents of Selectfluor ${ }^{\mathrm{TM}}$ (Table 2, conditions C).

Table 2 Synthesis of Fluoropyrazole and $1 H$-Difluoropyrazole Derivatives

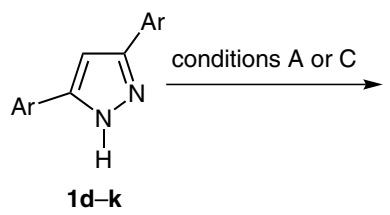<smiles>Fc1c(Br)n[nH]c1Br</smiles>

2d-k

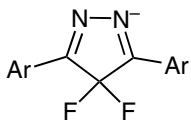

$3 a-h$

\begin{tabular}{|c|c|c|c|c|}
\hline Pyrazole 1 & $\mathrm{Ar}$ & Conditions $^{\mathrm{a}, \mathrm{b}}$ & $\begin{array}{l}\text { Fluoropyrazole } \\
\mathbf{2} \text {, yield (\%) }\end{array}$ & $\begin{array}{l}\text { Difluoropyra- } \\
\text { zole 3, yield (\%) }\end{array}$ \\
\hline 1d & $\mathrm{Ph}$ & $\begin{array}{l}A \\
C\end{array}$ & $\begin{array}{l}\text { 2d, } 45 \\
\text { 2d, } 23\end{array}$ & $\begin{array}{l}3 a, 21 \\
3 a, 52\end{array}$ \\
\hline $1 e$ & 4- $\mathrm{ClC}_{6} \mathrm{H}_{4}$ & $\begin{array}{l}A \\
C\end{array}$ & $\begin{array}{l}2 e, 31^{c} \\
2 e, 15^{c}\end{array}$ & $\begin{array}{l}3 b, 22 \\
3 b, 54\end{array}$ \\
\hline $1 f$ & $4-\mathrm{BrC}_{6} \mathrm{H}_{4}$ & $\begin{array}{l}\mathrm{A} \\
\mathrm{C}\end{array}$ & $\begin{array}{l}\mathbf{2 f}, 37^{c} \\
\mathbf{2 f}, 19^{c}\end{array}$ & $\begin{array}{l}3 c, 22 \\
3 c, 54\end{array}$ \\
\hline $1 \mathrm{~g}$ & $4-\mathrm{F}_{3} \mathrm{CC}_{6} \mathrm{H}_{4}$ & $\begin{array}{l}A \\
C\end{array}$ & $\begin{array}{l}\mathbf{2 g}, 36^{c} \\
\mathbf{2 g}, 20^{c}\end{array}$ & $\begin{array}{l}\text { 3d, } 51 \\
\text { 3d, } 33\end{array}$ \\
\hline $1 \mathrm{~h}$ & $3-\mathrm{F}_{3} \mathrm{CC}_{6} \mathrm{H}_{4}$ & $\begin{array}{l}A \\
C\end{array}$ & $\begin{array}{l}2 \mathrm{~h}, 41^{\mathrm{c}} \\
\mathbf{2 h}, 24^{\mathrm{c}}\end{array}$ & $\begin{array}{l}3 e, 20 \\
3 e, 44\end{array}$ \\
\hline $1 \mathbf{i}$ & 4- $\mathrm{MeOC}_{6} \mathrm{H}_{4}$ & $\begin{array}{l}\mathrm{A} \\
\mathrm{C}\end{array}$ & $\begin{array}{l}2 i, 45 \\
2 i, 20\end{array}$ & $\begin{array}{l}3 f, 27 \\
3 f, 45\end{array}$ \\
\hline $1 \mathbf{j}$ & 3- $\mathrm{MeOC}_{6} \mathrm{H}_{4}$ & $\begin{array}{l}A \\
C\end{array}$ & $\begin{array}{l}2 \mathrm{j}, 31 \\
2 \mathrm{j}, 24\end{array}$ & $\begin{array}{l}3 g, 24 \\
3 g, 43\end{array}$ \\
\hline $1 k$ & $2-\mathrm{MeOC}_{6} \mathrm{H}_{4}$ & $\begin{array}{l}A \\
C\end{array}$ & $\begin{array}{l}\mathbf{2 k}, 42 \\
\mathbf{2 k}, 17\end{array}$ & $\begin{array}{l}\text { 3h, } 23 \\
\text { 3h, } 49\end{array}$ \\
\hline
\end{tabular}

${ }^{a}$ Conditions A: Selectfluor ${ }^{\mathrm{TM}}$ ( 1 equiv), MW, $15 \mathrm{~min}, 90^{\circ} \mathrm{C}$.

${ }^{\mathrm{b}}$ Conditions C: Selectfluor ${ }^{\mathrm{TM}}$ ( 2 equiv), MW, 15 min, $90^{\circ} \mathrm{C}$.

c Products $\mathbf{2 e - h}$ could not be separated from starting materials $\mathbf{1 e}-\mathbf{h}$ respectively by column chromatography and yields were estimated by ${ }^{19} \mathrm{~F}$ NMR in these cases only.

In contrast, when 3,5-diarylpyrazoles $\mathbf{1 d - k}$ were reacted with fluorine gas, many fluorinated products were observed by ${ }^{19} \mathrm{~F}$ NMR analysis of the crude product mixture and no products could be isolated and purified. In these reactions, competing fluorination of the aromatic ring substituents occurs as determined by the observation of many signals in the aromatic region $\left(\delta_{\mathrm{F}}=-140\right.$ to $\left.-160 \mathrm{ppm}\right)$ of the ${ }^{19} \mathrm{~F}$ NMR spectra of the crude product mixture.

Difluorinated products $\mathbf{3 a}-\mathbf{h}$ were characterized by distinctive singlet resonances at approximately $\delta=-115 \mathrm{ppm}$ in their ${ }^{19} \mathrm{~F}$ NMR spectra and the structure of $\mathbf{3 f}$ was confirmed by X-ray crystallography (Figure 2). ${ }^{18}$ The difluori- 
nated pyrazole systems $\mathbf{3}$ are a novel class of fluorinated compounds although the corresponding dichlorinated systems have been reported and their use in Diels-Alder reactions has been explored. ${ }^{19}$

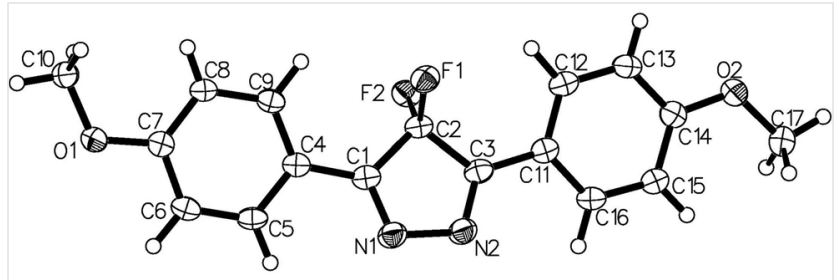

Figure 2 Molecular structure of $\mathbf{3 f}$

Initial fluorination of pyrazole derivatives occurs selectively at the 4-position consistent with an electrophilic aromatic substitution process (Scheme 1) and further electrophilic fluorination reaction occurs at the same site to give a difluorinated salt $\mathbf{4}$ as an intermediate. Deprotonation on workup gives the observed 4,4-difluoro-1H pyrazole product 3 .

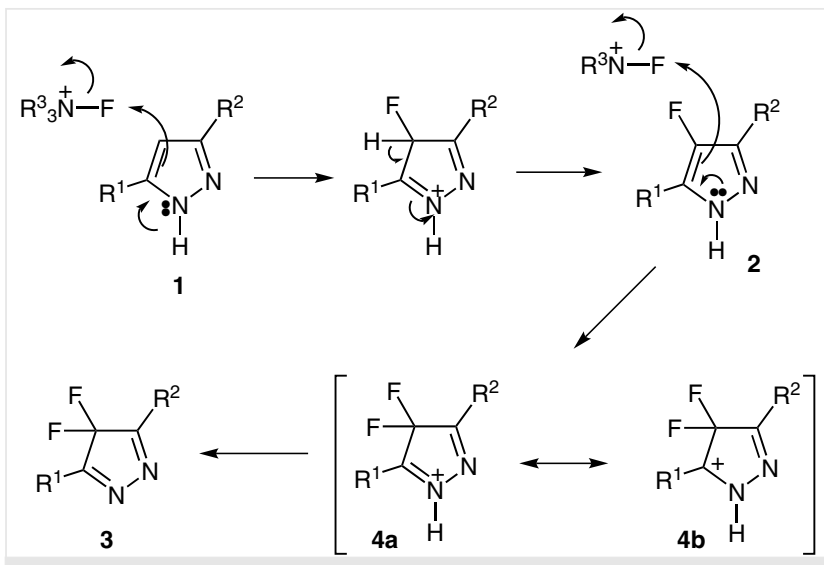

Scheme 1 Fluorination of pyrazole derivatives $\mathbf{2}$ and $\mathbf{3}$

The outcome is consistent with the intermediate carbocation $\mathbf{4 b}$ being stabilized by the adjacent phenyl groups $\left(\mathrm{R}^{1}\right.$ = aryl; Scheme 1) allowing difluorination to proceed as observed for 3,5-diarlpyrazole substrates.

For reaction with dibrominated system 11, the hydroxypyrazoline $\mathbf{5}$ could be isolated albeit in low yield and, in some analogous reactions, ${ }^{19} \mathrm{~F}$ NMR analysis indicated the presence of hydroxylated systems consistent with $\mathbf{5}$ in crude product mixtures (Scheme 2). This minor product is formed by reaction of water with intermediate salt $\mathbf{4}$ in reaction workup, consistent with the mechanism shown in Scheme 1 and related reactions involving other halogenated $4 H$-pyrazoles. ${ }^{20}$ The hydroxypyrazoline product $\mathbf{5}$ could be identified by the presence of an $\mathrm{AB}$ system, with an appropriate $J_{\mathrm{AB}}=128 \mathrm{~Hz}$ coupling constant, in the ${ }^{19} \mathrm{~F}$ NMR spectrum.

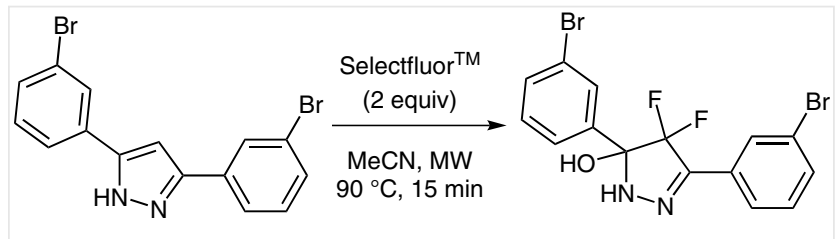

11

5, $24 \%$

Scheme 2 Hydoxylated pyrazoline $\mathbf{5}$

In conclusion, a method for the synthesis of unusual 4,4-difluoro- $1 H$-pyrazole systems $\mathbf{3}^{21}$ has been established using shelf-stable, readily handled Selectfluor ${ }^{\mathrm{TM}}$ as the electrophilic fluorinating agent.

\section{Acknowledgment}

We thank Dr. D. S. Yufit (Durham University) for X-ray crystallographic structural analysis.

\section{Supporting Information}

Supporting information for this article is available online at http://dx.doi.org/10.1055/s-0034-1378915.

\section{References and Notes}

(1) (a) O'Hagan, D. Chem. Soc. Rev. 2008, 37, 308. (b) Purser, S.; Moore, P. R.; Swallow, S.; Gouverneur, V. Chem. Soc. Rev. 2008, 37, 320. (c) Hagmann, W. K. J. Med. Chem. 2008, 51, 4359. (d) Muller, K.; Faeh, C.; Diedrich, F. Science 2007, 317, 1881. (e) Fluorine in Medicinal Chemistry and Chemical Biology; Ojima, I., Ed.; Wiley-Blackwell: Oxford, 2009.

(2) (a) For FY 2011 Innovative Drug Approvals, see: http://www.fda.gov/AboutFDA/ReportsManualsForms/Reports/ucm276385.htm. (b) Ilardi, E. A.; Vitaku, E.; Njardarson, J. T. J. Med. Chem. 2014, 57, 2832.

(3) Petrov, V. A. Fluorinated Heterocyclic Compounds: Synthesis, Chemistry and Applications; John Wiley and Sons: New York, 2009.

(4) Horiuchi, Y.; Nunami, N.; Tatamidani, H.; Ohata, E. PCT Int. Appl WO2009020137 AI20090212, 2009.

(5) Dressen, D.; Garofalo, A. W.; Hawkinson, J.; Hom, D.; Jagodzinski, J.; Marugg, J. L.; Neitzel, M. L.; Pleiss, M. A.; Szoke, B.; Tung, J. S.; Wone, D. W. G.; Wu, J.; Zhang, H. J. Med. Chem. 2007, 50, 5161.

(6) Large, M. S. Eur. Pat. Appl EP61318 A219820929, 1982.

(7) Ohata, S.; Kato, K.; Toriyabe, K.; Ito, Y.; Hamaguchi, R.; Nakano, Y. PCT Int. Pat. Appl WO2009051245 AI20090423, 2009.

(8) Surmont, R.; Verniest, G.; DeSchrijver, M.; Thuring, J. W.; ten Holte, P.; Derouse, F.; De Kimpe, N. J. Org. Chem. 2011, 76, 4105.

(9) Surmont, R.; Verniest, G.; De Kimpe, N. Org. Lett. 2010, 12, 4648.

(10) Qian, J.; Liu, Y.; Zhu, J.; Jiang, B.; Xu, Z. Org. Lett. 2011, 13, 4220.

(11) (a) Sloop, J. C.; Bumgardner, C. L.; Loehle, W. D. J. Fluorine Chem. 2002, 118, 135. (b) Breen, J. R.; Sandford, G.; Yufit, D. S.; Howard, J. A. K.; Fray, J.; Patel, B. Beilstein J. Org. Chem. 2011, 7, 1048.

(12) Katoch-Rouse, R.; Horti, A. G. J. Labelled Compd. Radiopharm. 2003, 46, 93.

(13) Fabra, F.; Vilarrasa, J. J. Heterocycl. Chem. 1978, 15, 1447. 
(14) Bentley, J.; Biagetti, M.; Di Fabio, R.; Genski, T.; Guery, S.; Kopf, S. R.; Leslie, C. P.; Mazzali, A.; Meletto, S.; Pizzi, D. A.; Sabbatini, F. M.; Seri, C. PCT Int. Pat. Appl WO 2008092888 A120080807, 2008.

(15) Sloop, J. C.; Jackson, J. L.; Schmidt, R. D. Heteroat. Chem. 2009, $20,341$.

(16) (a) Chambers, R. D.; Parsons, M.; Sandford, G.; Moilliet, J. S. J. Chem. Soc., Perkin Trans 1 2002, 2190. (b) Sandford, G. J. Fluorine Chem. 2007, 128, 90. (c) McPake, C. B.; Sandford, G. Org. Process Res. Dev. 2012, 16, 844.

(17) Grandberg, I. I.; Kost, A. N. Adv. Heterocycl. Chem. 1966, 6, 347.

(18) X-ray crystallographic data has been deposited at the Cambridge Crystallographic Data Centre as CCDC 1016969-1016970.

(19) Adam, W.; Ammon, H.; Nau, W. M.; Peters, K.J. Org. Chem. 1994, 59, 7067.

(20) Hansen, J.; Kim, Y.; Griswold, L.; Hoelle, G.; Taylor, D.; Vietti, D. J. Org. Chem. 1980, 45, 76.

(21) Typical Procedure (Conditions A); 4-Fluoro-3,5-diphenyl-1Hpyrazole (2d) and 4,4-Difluoro-3,5-diphenyl-4H-pyrazole (3a): 3,5-Diphenyl-1H-pyrazole $(0.30 \mathrm{~g}, 1.36 \mathrm{mmol})$ and Selectfluor $^{\mathrm{TM}}(0.482 \mathrm{~g}, 1.36 \mathrm{mmol})$ were dissolved in $\mathrm{MeCN}(5 \mathrm{~mL})$ and the mixture was heated by microwave irradiation for $15 \mathrm{~min}$ at
$90{ }^{\circ} \mathrm{C}$. The mixture was then extracted with $\mathrm{CH}_{2} \mathrm{Cl}_{2}(3 \times 50 \mathrm{~mL})$ and washed with $\mathrm{NaHCO}_{3}(30 \mathrm{~mL})$ and $\mathrm{H}_{2} \mathrm{O}(30 \mathrm{~mL})$. The combined extracts were dried $\left(\mathrm{MgSO}_{4}\right)$ and evaporated. Column chromatography on silica gel using hexane and EtOAc (1:1) as the eluent, gave 4-fluoro-3,5-diphenyl- $1 \mathrm{H}$-pyrazole $(0.135 \mathrm{~g}$, $45 \%)$ as pale yellow crystals; mp $185-188^{\circ} \mathrm{C} .{ }^{1} \mathrm{H}$ NMR $(400 \mathrm{MHz}$, $\left.\mathrm{CDCl}_{3}\right): \delta=7.41-7.47(\mathrm{~m}, 2 \mathrm{H}, 4-\mathrm{H}), 7.48-7.51(\mathrm{~m}, 4 \mathrm{H}, 3-\mathrm{H})$, 7.77-7.80 (m, $4 \mathrm{H}, 2-\mathrm{H}), 10.3$ (br s, $1 \mathrm{H}, \mathrm{NH}){ }^{13} \mathrm{C} \mathrm{NMR}(126 \mathrm{MHz}$, $\left.\mathrm{CDCl}_{3}\right): \delta=128.2(\mathrm{Ar}), 129.0(\mathrm{Ar}), 129.3(\mathrm{Ar}), 131.1\left(\mathrm{~d},{ }^{2} J_{\mathrm{CF}}=15.0\right.$ $\mathrm{Hz}, \mathrm{C}-3), 140.0\left(\mathrm{~d},{ }^{1} J_{\mathrm{CF}}=226.6 \mathrm{~Hz}, \mathrm{C}-4\right), 148.7(\mathrm{Ar}) .{ }^{19} \mathrm{~F}$ NMR $(376$ $\left.\mathrm{MHz}, \mathrm{CDCl}_{3}\right): \delta=-174.3(\mathrm{~s})$. MS: $m / z\left(\%, \mathrm{EI}^{+}\right)=237.9(100)[\mathrm{M}]^{+}$, 107.8 (43), 76.9 (40). HRMS: $m / z$ [M $+\mathrm{H}]^{+}$calcd for $\mathrm{C}_{15} \mathrm{H}_{12} \mathrm{FN}_{2}$ : 239.0983; found: 239.0972. 4,4-Difluoro-3,5-diphenyl-4H-pyrazole (3a): obtained as yellow crystals $(0.122 \mathrm{~g}, 21 \%)$; mp $105-$ $107{ }^{\circ} \mathrm{C} .{ }^{1} \mathrm{H}$ NMR $\left(400 \mathrm{MHz}, \mathrm{CDCl}_{3}\right): \delta=7.44-7.67(\mathrm{~m}, 6 \mathrm{H}, \mathrm{ArH})$, 8.06-8.15 (m, $4 \mathrm{H}, \mathrm{ArH}) .{ }^{13} \mathrm{C}$ NMR (126 MHz, $\left.\mathrm{CDCl}_{3}\right): \delta=125.4$ (Ar), $125.6\left(\mathrm{t},{ }^{1} J_{\mathrm{CF}}=267.5 \mathrm{~Hz}, \mathrm{CF}_{2}\right), 128.3(\mathrm{Ar}), 129.5(\mathrm{Ar}), 133.1$ (Ar), $162.1\left(\mathrm{t},{ }^{2} \mathrm{~J}_{\mathrm{CF}}=23.1 \mathrm{~Hz}, \mathrm{C}-2\right) \cdot{ }^{19} \mathrm{~F}$ NMR $\left(376 \mathrm{MHz}, \mathrm{CDCl}_{3}\right): \delta=$ -116.3 (s). MS: $m / z\left(\%, \mathrm{El}^{+}\right)=256.1(100)[\mathrm{M}]^{+}, 153.0(45), 103.1$ (99), 77.1 (36). HRMS: $m / z[\mathrm{M}+\mathrm{H}]^{+}$calcd for $\mathrm{C}_{15} \mathrm{H}_{11} \mathrm{~F}_{2} \mathrm{~N}_{2}$ : 257.0890; found: 257.0894 . 